\title{
summary
}

\section{No consensus on definition of early childhood caries}

Ismail Al, Sohn W. A systematic review of clinical diagnostic criteria of early childhood caries. J Publ Health Dent 1999; 59:171-191

Question: Are there clear agreed definitions for early or severe childhood caries?

Objective To review case definitions and clinical diagnostic criteria for early childhood caries (ECC) and severe ECC (S-ECC).

Data sources MEDLINE 1966-1998, references from three previous reviews and one unpublished report.

Study selection Studies were selected if they included children aged 1-5 years of age, described diagnostic criteria or case-definitions of S-ECC, and were in English.

Results A total of 71 studies collected population data and the rest described children seen in health or dental clinics (see Table 1). Fiftynine studies did not report on calibration of examiners, but reliability of examiners was reported by 19 studies. Cavitation was the most common diagnostic criterion (44 studies). Whereas 22 studies reported no diagnostic criterion, 27 used a criterion of one or more dmf maxillary incisors, 23 used two or more, and nine used three or more dmf maxillary incisors for their diagnosis. The most frequently used names for S-ECC were "nursing caries" (23 studies), "babybottle tooth decay" (14 studies) and rampant caries (nine studies). Studies published in the last 2 years estimated that 1963\% of examined children had S-ECC.
Table 1 Studies on early childhood caries included in report

\begin{tabular}{lc}
\hline Type of studies & Studies included $(\mathrm{n})$ \\
\hline Cross-sectional surveys & 81 \\
Case-control studies & 7 \\
Controlled clinical trials & 2 \\
Cohort studies/field trials & 4 \\
Total & 94 \\
\hline
\end{tabular}

Conclusions There is wide variation in the diagnostic criteria for ECC and case-definition for S-ECC. A consensus is needed for research and to evaluate preventive interventions.
Evidence-Based
Dentistry
(2002)
3, 75
doi:10.1038/
sj.ebd.6400106

Address for reprints: Professor Amid Ismail, Department of Cariology, Restorative Sciences and Endodontics, D2347, School of Dentistry, University of Michigan, 1011 N. University, Ann Arbor MI 481091078, USA. E-mail: ismailai@umich.edu

\section{Commentary}

In recent years systematic reviews and meta-analyses of published research have begun to change mindsets in many clinical disciplines. Dogma has been challenged and information on which the dogma might be based has been questioned. Not surprisingly weakness in such information has been exposed. Ismail and Sohn have done cariology a great service through their systematic review of ECC. For too long in my opinion have narrative reviews, mostly highly selective of articles for inclusion, dominated cariology. The current study has been carried out well according to standard methods for systematic reviews. It is remarkably comprehensive even if, as the authors modestly admit, it is not totally comprehensive. The logic of the article is clear and their text is easily understood thanks to the excellent tables.
The systematic review shows that cross-sectional studies dominate with few longitudinal studies in the published literature, and for good reason-longitudinal caries studies are difficult to do, expensive and timeconsuming. Cross-sectional information is very useful, however. It may be widely applicable provided that clear diagnostic criteria have been used, there is convincing attention to study population selection and calibration of examiners, as well as maintenance of diagnostic standards. Sadly, Ismail and Sohn have shown how inconsistent and weak so many articles have been; indeed 22 out of the 94 studies did not even describe what diagnostic criteria were used. I have found the same thing in a systematic review of caries in Africa. ${ }^{1}$ There, I lamented how simple World Health Organization caries diagnostic criteria have been ignored by so many in spite of being available now for over 30 years.

I agree completely that there is a need for an internationally accepted definition for ECC and S-ECC. Caries rates reports using such definitions would be of wide benefit in service planning and disease surveillance. Investigators would not be limited by these definitions since they could be used in all caries rates studies in addition to other measurements.

1. Cleaton-Jones PE, Fatti LP. Dental caries trends in Africa. Community Dent Oral Epidemiol 1999; 27:316-320.

Peter Cleaton-Jones Dental Research Institute, University of Witwatersrand, Johannesburg, South Africa 